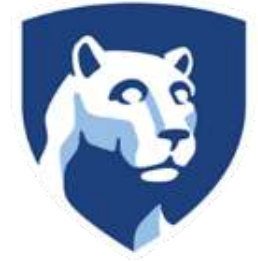

PennState

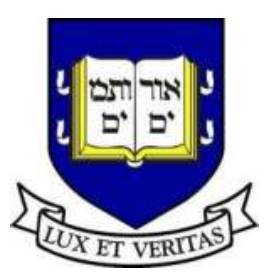

Yale

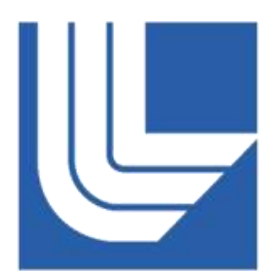

LLNL

\title{
A numerical study on the sooting tendencies of bio-derived fuels for spark-ignition engines
}

Hyunguk Kwon', Simon Lapointe'2, Kuiwen Zhang², Scott W. Wagnon², William J. Pitz', Junqing Zhu ${ }^{3}$, Charles S. McEnally ${ }^{3}$, Lisa D. Pfefferle ${ }^{3}$, Yuan Xuan ${ }^{4}$

'Department of Chemical Engineering, Pennsylvania State University ${ }^{2}$ Materials Science Division, Lawrence Livermore National Laboratory ${ }^{3}$ Department of Chemical and Environmental Engineering, Yale University ${ }^{4}$ Department of Mechanical Engineering, Pennsylvania State University 
Energy Sources Fossil energy (petroleum, coal, natural gas)

- Biofuel: fuels produced from biomass that can replace petroleum fuels

- Advantages ${ }^{[1]}$
- Various source material
- Sustainability
- Reduction of greenhouse gas emissions

- Barriers to the realization of biofuels' implementation target ${ }^{[2]}$
- Shortage of feedstocks
- Higher costs than traditional fuels
- Variations in performance 


\section{Identification of Promising Bio-derived Fuels}

\section{$\square$ Co-Optimization of Fuels \& Engines (Co-Optima)}

$\rightarrow$ Fuels intended to enable high-efficiency, low-emissions engines

$\rightarrow$ Fuel screening using a tiered process

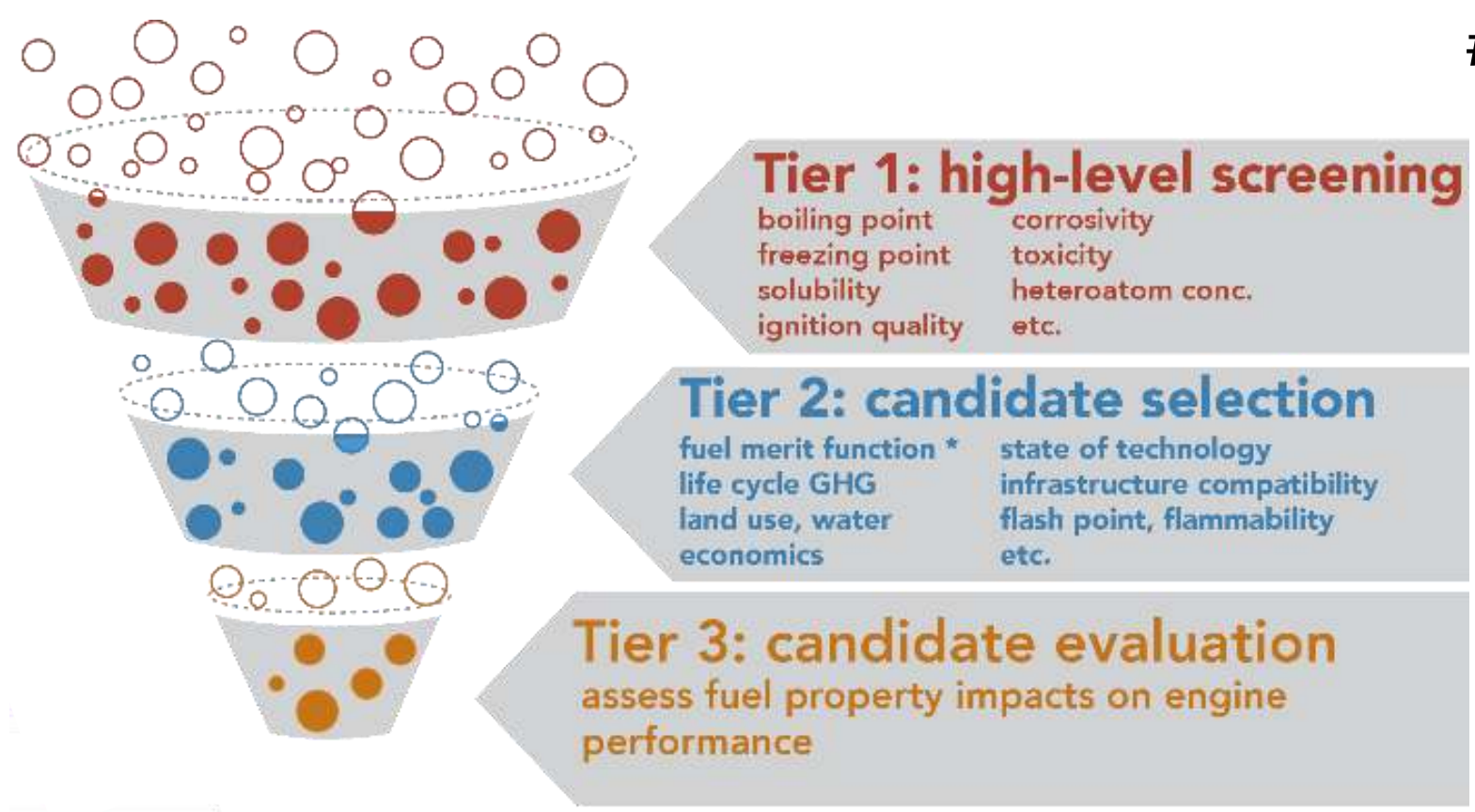

\# Blendstocks \# Chemical families

(screening

$>470$

heteroatom conc.

\section{Tier 2: candidate selection}

fuel merit function * state of technology

41

10

8 


\section{Selected Fuels}

Alcohol

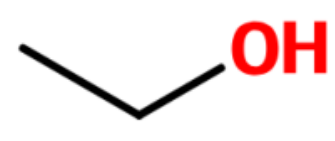

ethanol

Ketone

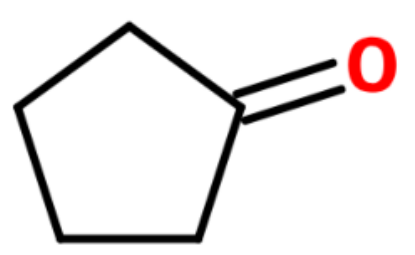

cyclopentanone

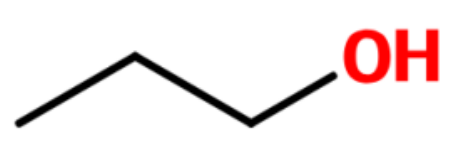

$n$-propanol

Alkene

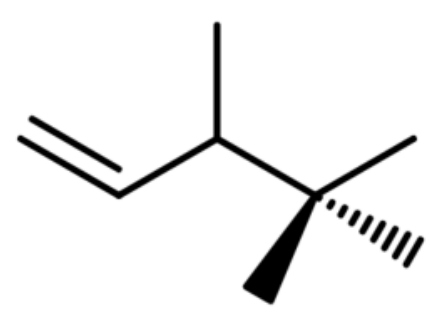

diisobutylene

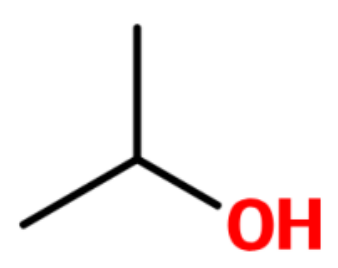

i-propanol

Furan mixture

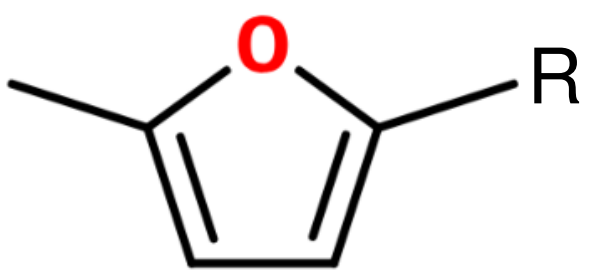

$\mathrm{R}=\mathrm{H},-\mathrm{CH}_{3}$

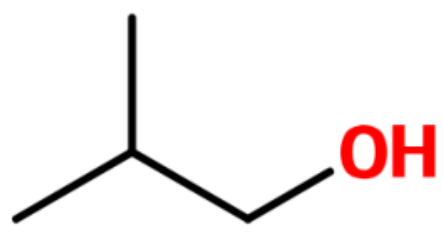

i-butanol

Aromatic

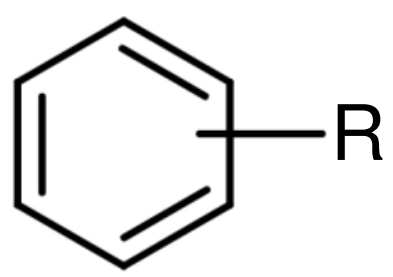

- Potential to increase the efficiency of SI engines, meet key fuel-quality requirements, and be commercially available by 2025-2030

- $\quad$ YSI has been measured to characterize sooting tendencies. 


\section{Objectives}

(1) Predict YSIs of the promising blendstocks using numerical simulations

- YSI has never been computed for those fuels

- Efficiently predict YSIs using a perturbation-based approach

- Validate the ability of a kinetic model

(2) Quantify the effects of chemical kinetic uncertainties on YSI predictions

- Quantify impact of PAH growth reactions on YSI

- Identify the most sensitive reactions to YSI predictions

- Quantify rate constant uncertainties on YSI Predictions 


\section{Measurement of Sooting Tendency}

$\square$ Yield Sooting Index (YSI) ${ }^{[1]}$

- It defines sooting tendency based on soot yield

- Measure of max soot volume fraction $\left(f_{v, \max }\right)$

- YSI is defined as a linear function of $\mathrm{f}_{\mathrm{v}, \max }$

$$
\mathrm{YSI}(\text { dopant })=\mathrm{A} \times \mathrm{f}_{\mathrm{v}, \text { max }}(\text { dopant })+\mathrm{B}
$$

$\mathrm{YSI}=36.0$ for $n$-heptane,

$\mathrm{YSI}=170.9$ for toluene

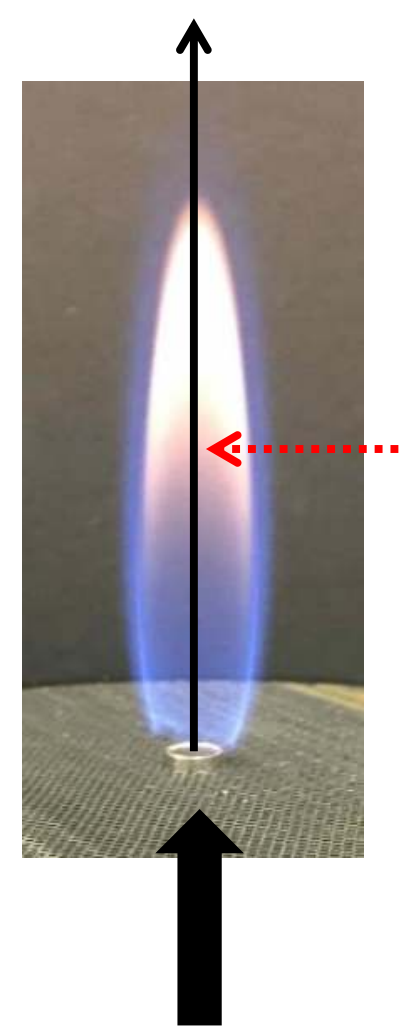

Measure $f_{v, \max }$ along the centerline

Test compound, $0.1 \%$ by mole Methane, $40 \%$ by mole Nitrogen, balance

[Yale co-flow burner] 


\section{YSI is easy to be modeled computationally}

- Only one 2D flame simulation is required to calculate YSI

- YSI computation for each test compound is efficient (1D simulations along the flame centerline)

- Agreement with experimental YSIs for aliphatic, aromatic compounds, surrogate fuels

Flame centerline
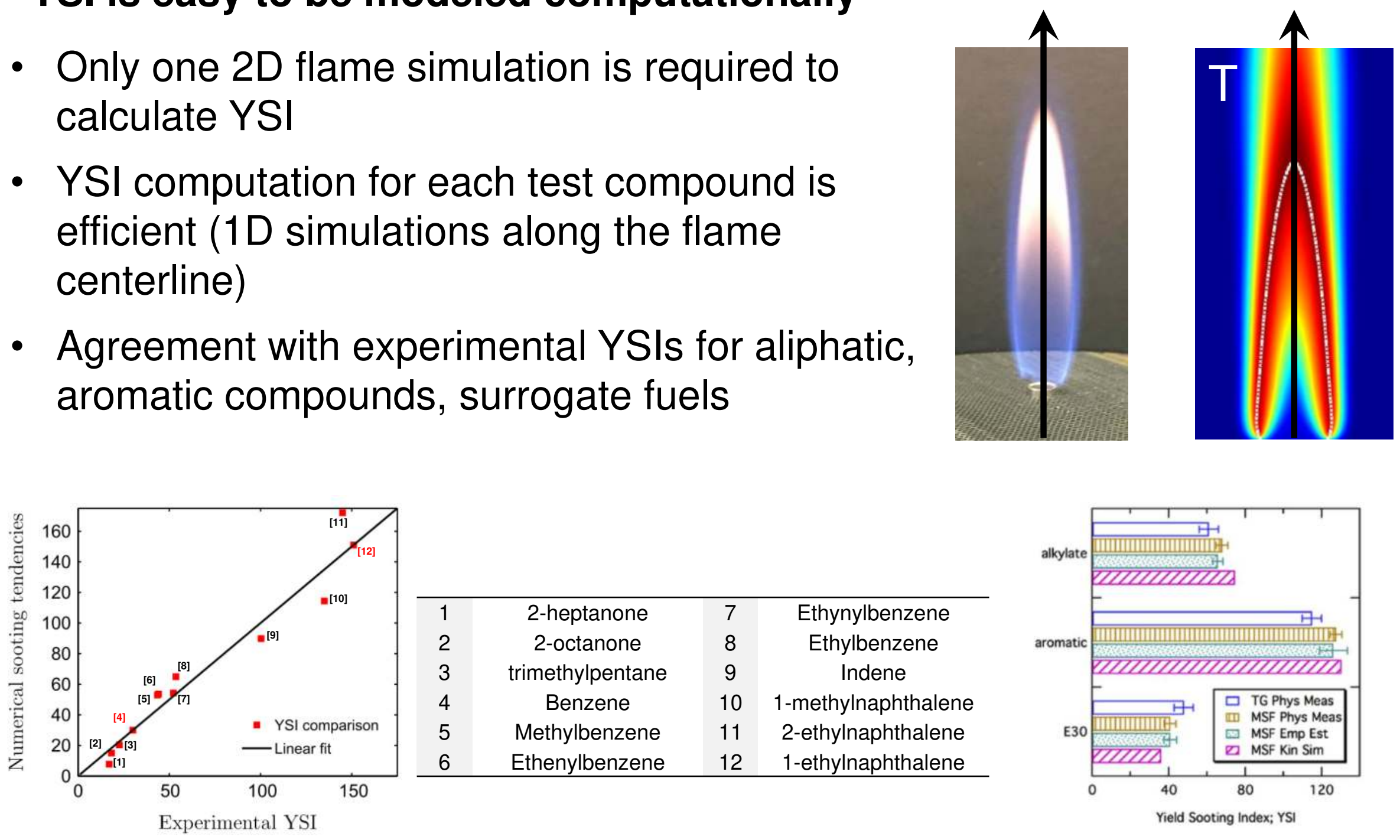

\begin{tabular}{c|c|c|c}
\hline 1 & 2-heptanone & 7 & Ethynylbenzene \\
2 & 2-octanone & 8 & Ethylbenzene \\
3 & trimethylpentane & 9 & Indene \\
4 & Benzene & 10 & 1-methylnaphthalene \\
5 & Methylbenzene & 11 & 2-ethylnaphthalene \\
6 & Ethenylbenzene & 12 & 1-ethylnaphthalene \\
\hline
\end{tabular}

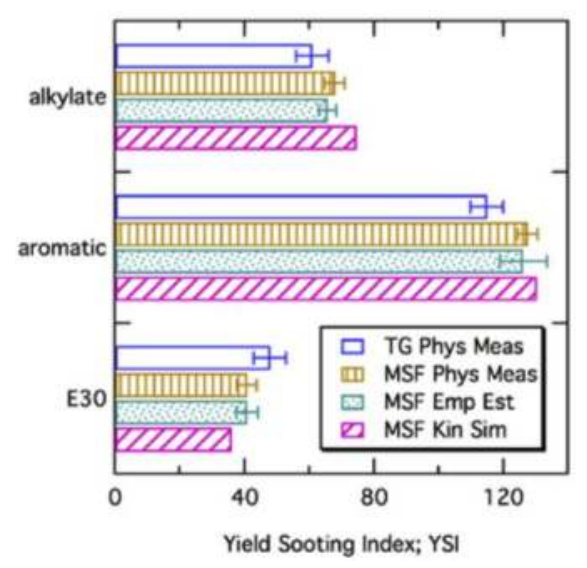

[1] Y. Xuan, G. Blanquart, Combust. Flame 160 (2013) 1657-1666

[2] C.S. McEnally et al., Proc. Combust. Inst. 37 (2019) 961-968 


\section{Co-Optima Kinetic Model}

Co-Optima chemical model has been developed for transportation fuels by

LLNL, NUI Galway, and KAUST teams[1]

- Co-Optima model can handle Co-Optima fuels

$\mathrm{C}_{1}-\mathrm{C}_{5}$ alcohols

Ester (methyl acetate, ethyl acetate, methyl butanoate)

Ketone (cyclopentanone, 2-butanone)

Furan (2-methylfuran, 2,5-dimethylfuran)

Alkene (di-isobutylene)

- Validated against ignition and flame propagation for a broad range of $\Phi, T, P$

- A reduced model generated by removing the low temperature chemistry

- 1172 species, 7517 reactions

- Hydrocarbon growth up to naphthalene 


\section{List of Co-Optima Fuels Considered}

\begin{tabular}{|c|c|c|c|c|c|}
\hline$\#$ & Name & Family group & $\#$ & Name & Family group \\
\hline 1 & $\begin{array}{l}\text { Methanol } \\
\left(\mathrm{CH}_{4} \mathrm{O}\right)\end{array}$ & Alcohols & 11 & $\begin{array}{l}\text { 2-methyl-1-butanol } \\
\left(\mathrm{C}_{5} \mathrm{H}_{12} \mathrm{O}\right)\end{array}$ & Alcohols \\
\hline 2 & $\begin{array}{l}\text { ethanol } \\
\left(\mathrm{C}_{2} \mathrm{H}_{6} \mathrm{O}\right) \\
\end{array}$ & Alcohols & 12 & $\begin{array}{c}\text { methyl acetate } \\
\qquad\left(\mathrm{C}_{3} \mathrm{H}_{6} \mathrm{O}_{2}\right)\end{array}$ & Esters \\
\hline 3 & $\begin{array}{c}\text { n-propanol } \\
\left(\mathrm{C}_{3} \mathrm{H}_{8} \mathrm{O}\right) \\
\end{array}$ & Alcohols & 13 & $\begin{array}{l}\text { ethyl acetate } \\
\left(\mathrm{C}_{4} \mathrm{H}_{8} \mathrm{O}_{2}\right) \\
\end{array}$ & Esters \\
\hline 4 & $\begin{array}{l}\text { iso-propanol } \\
\left(\mathrm{C}_{3} \mathrm{H}_{8} \mathrm{O}\right) \\
\end{array}$ & Alcohols & 14 & $\begin{array}{l}\text { methyl butanoate } \\
\qquad\left(\mathrm{C}_{5} \mathrm{H}_{10} \mathrm{O}_{2}\right)\end{array}$ & Esters \\
\hline 5 & $\begin{array}{l}n \text {-butanol } \\
\left(\mathrm{C}_{4} \mathrm{H}_{10} \mathrm{O}\right) \\
\end{array}$ & Alcohols & 15 & $\begin{array}{c}\text { 2-butanone } \\
\left(\mathrm{C}_{4} \mathrm{H}_{8} \mathrm{O}\right) \\
\end{array}$ & Ketones \\
\hline 6 & $\begin{array}{l}\text { 2-butanol } \\
\left(\mathrm{C}_{4} \mathrm{H}_{10} \mathrm{O}\right) \\
\end{array}$ & Alcohols & 16 & $\begin{array}{c}\text { cyclopentanone } \\
\left(\mathrm{C}_{5} \mathrm{H}_{8} \mathrm{O}\right)\end{array}$ & Ketones \\
\hline 7 & $\begin{array}{l}\text { 2-methyl-1-propanol } \\
\left(\mathrm{C}_{4} \mathrm{H}_{10} \mathrm{O}\right)\end{array}$ & Alcohols & 17 & $\begin{array}{l}\text { 2-methylfuran } \\
\left(\mathrm{C}_{5} \mathrm{H}_{6} \mathrm{O}\right)\end{array}$ & Furans \\
\hline 8 & $\begin{array}{l}\text { tert-butanol } \\
\left(\mathrm{C}_{4} \mathrm{H}_{10} \mathrm{O}\right)\end{array}$ & Alcohols & 18 & $\begin{array}{l}\text { 2,5-dimethylfuran } \\
\left(\mathrm{C}_{6} \mathrm{H}_{8} \mathrm{O}\right)\end{array}$ & Furans \\
\hline 9 & $\begin{array}{c}n \text {-pentanol } \\
\left(\mathrm{C}_{5} \mathrm{H}_{12} \mathrm{O}\right)\end{array}$ & Alcohols & 19 & $\begin{array}{c}\text { 2,4,4-trimethyl-1-pent } \\
\text { ene }\left(\mathrm{C}_{8} \mathrm{H}_{16}\right)\end{array}$ & Alkenes \\
\hline 10 & $\begin{array}{l}\text { 3-methyl-1-butanol } \\
\left(\mathrm{C}_{5} \mathrm{H}_{12} \mathrm{O}\right)\end{array}$ & Alcohols & 20 & $\begin{array}{c}\text { 2,4,4-trimethyl-2-pent } \\
\text { ene }\left(\mathrm{C}_{8} \mathrm{H}_{16}\right)\end{array}$ & Alkenes \\
\hline
\end{tabular}




\section{YSI of Co-Optima Fuels}

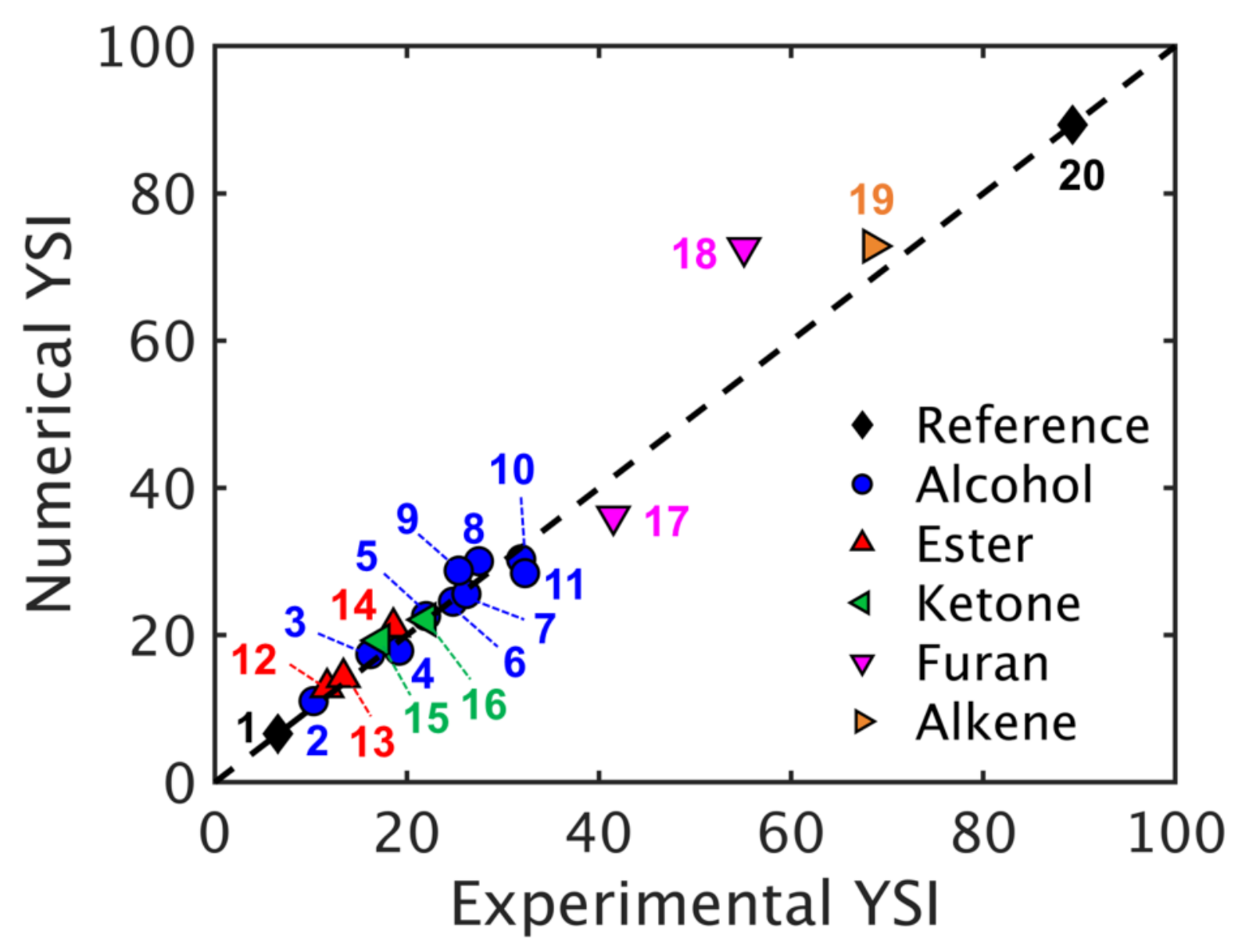

Reference fuels:

- 2,4,4-trimethyl-2-pentene (the most sooting fuel)

- methanol (the least sooting fuel) 


\section{YSI of Co-Optima Fuels}

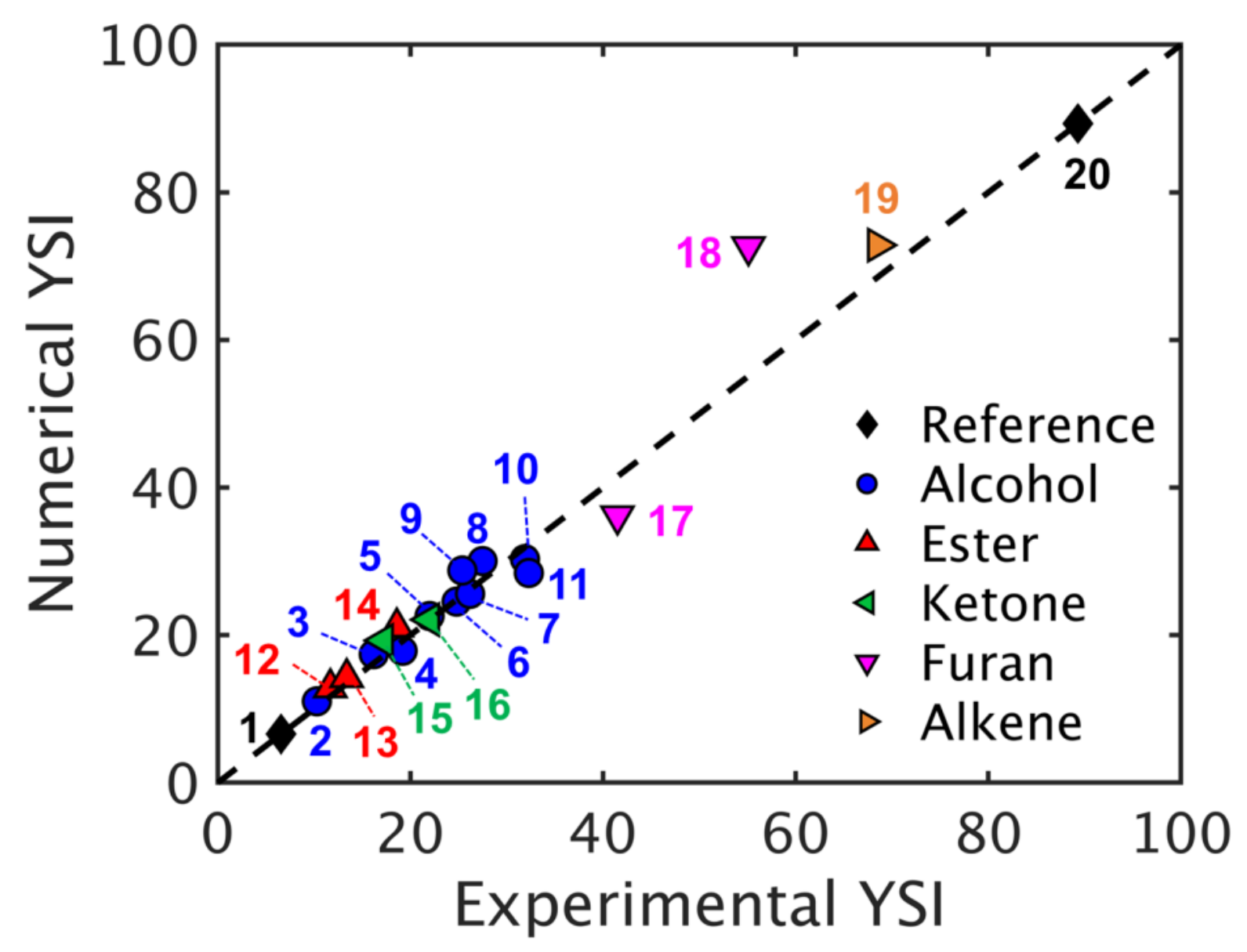

- Good agreement for most species, worst prediction for 25DMF - Furans and alkenes are sootiest

- Alcohols and esters and ketones are least sooty 


\section{Impact of PAH Growth Reactions on YSI}

Co-Optima Model:

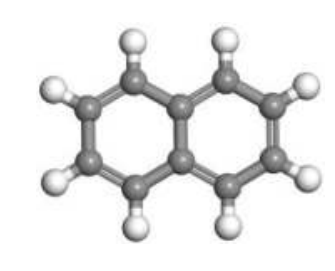

naphthalene

Expanded Model:

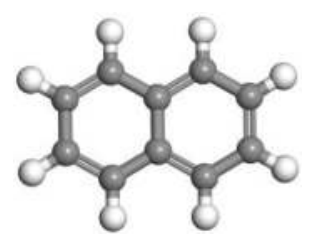

naphthalene
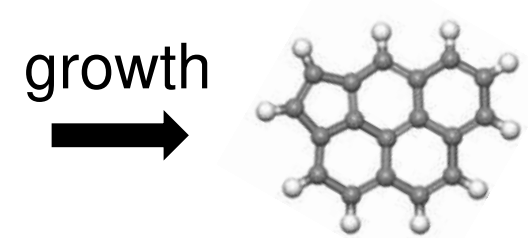

cyclopenta[cd]pyrene $]$
Polycyclic Aromatic Hydrocarbons

(PAHs)

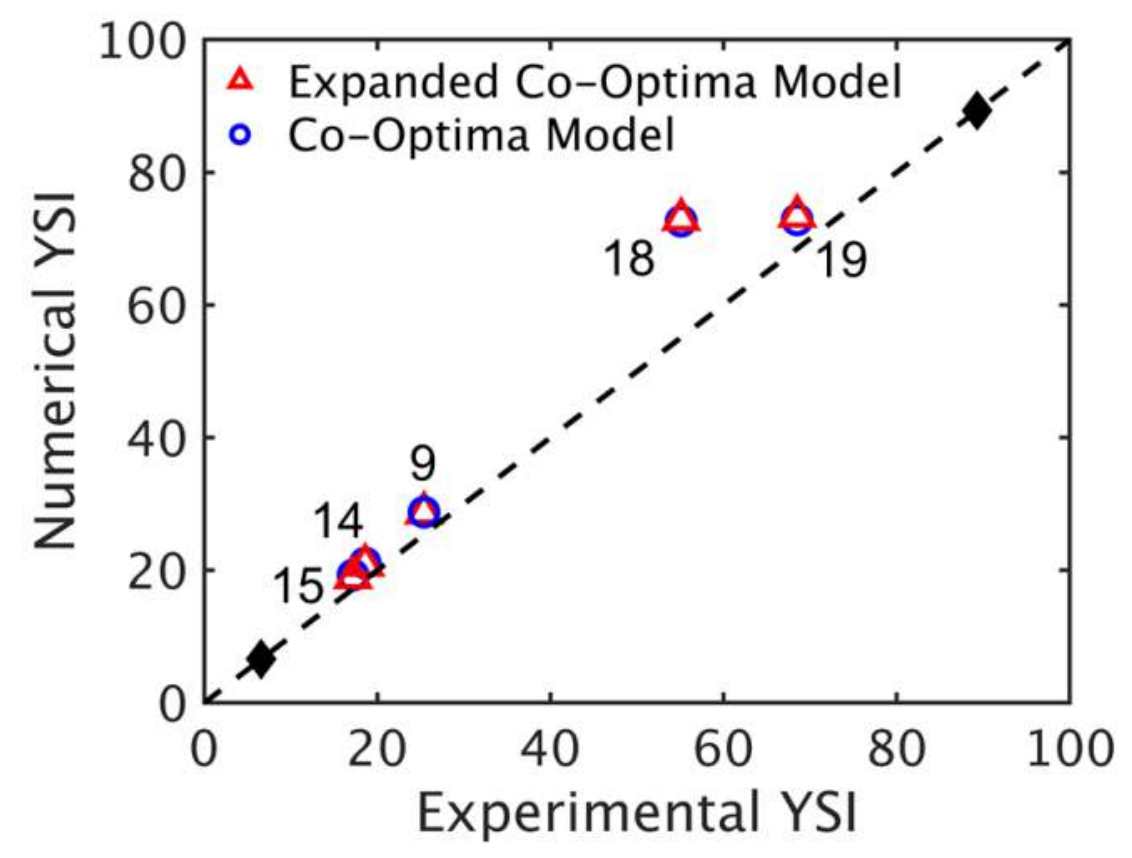

- Two different PAH sub-mechanisms agree to within $1.8 \%$

- Soot yield increases when using the Expanded Model, but the effects are canceled out when converting it to a relative sooting tendency (YSI) 


\section{Reaction Sensitivity Analysis of YSI}

\section{$\square$ Regular sensitivity analysis in combustion chemistry}

- Calculate sensitivity coefficients $(S)$ pertinent to a quantity of interest

- Reveals which are the main control parameters in the chemical model

This approach cannot be directly used in YSI

\section{$\square$ Sensitivity analysis in YSI}

- Calculate sensitivity coefficients in doped and undoped flames

- Calculate relative sensitivity coefficients: $S_{\text {rel }, j}=\left(S_{\text {doped }, j}-S_{\text {undoped }, j}\right) /\left(\left|S_{\text {undoped }, j}\right|+\varepsilon\right)$

- List of sensitive reactions in 2,5-DMF doped flame

\begin{tabular}{ccc}
\hline & Reactions & $\boldsymbol{S}_{\boldsymbol{r e l}, \boldsymbol{j}}$ \\
\hline $\mathrm{R} 1$ & 2,5-DMF $\rightarrow \mathrm{CH}_{3} \mathrm{CHCCH}(\mathrm{CO}) \mathrm{CH}_{3}$ & $-6.42 \mathrm{E}+06$ \\
$\mathrm{R} 2$ & 2,5-DMF $+\mathrm{CH}_{3} \rightarrow \mathrm{DMF} 252 \mathrm{~J}+\mathrm{CH}_{4}$ & $6.18 \mathrm{E}+06$ \\
$\mathrm{R} 3$ & $2,5-\mathrm{DMF}(+\mathrm{M}) \rightarrow \mathrm{DMF} 252 \mathrm{~J}+\mathrm{H}(+\mathrm{M})$ & $4.18 \mathrm{E}+06$ \\
$\mathrm{R} 4$ & $2,5-\mathrm{DMF}+\mathrm{H} \rightarrow \mathrm{DMF} 252 \mathrm{~J}+\mathrm{H}_{2}$ & $3.86 \mathrm{E}+06$ \\
$\mathrm{R} 5$ & $2,5-\mathrm{DMF}+\mathrm{H} \rightarrow 2-\mathrm{MF}+\mathrm{CH}_{3}$ & $-2.33 \mathrm{E}+06$ \\
$\mathrm{R} 6$ & $2,5-\mathrm{DMF}+\mathrm{H} \rightarrow 1,3-\mathrm{C}_{4} \mathrm{H}_{6}+\mathrm{CH}_{3} \mathrm{CO}$ & $-2.14 \mathrm{E}+06$ \\
\hline
\end{tabular}




\section{Reaction Flux Analysis in 2,5-DMF}

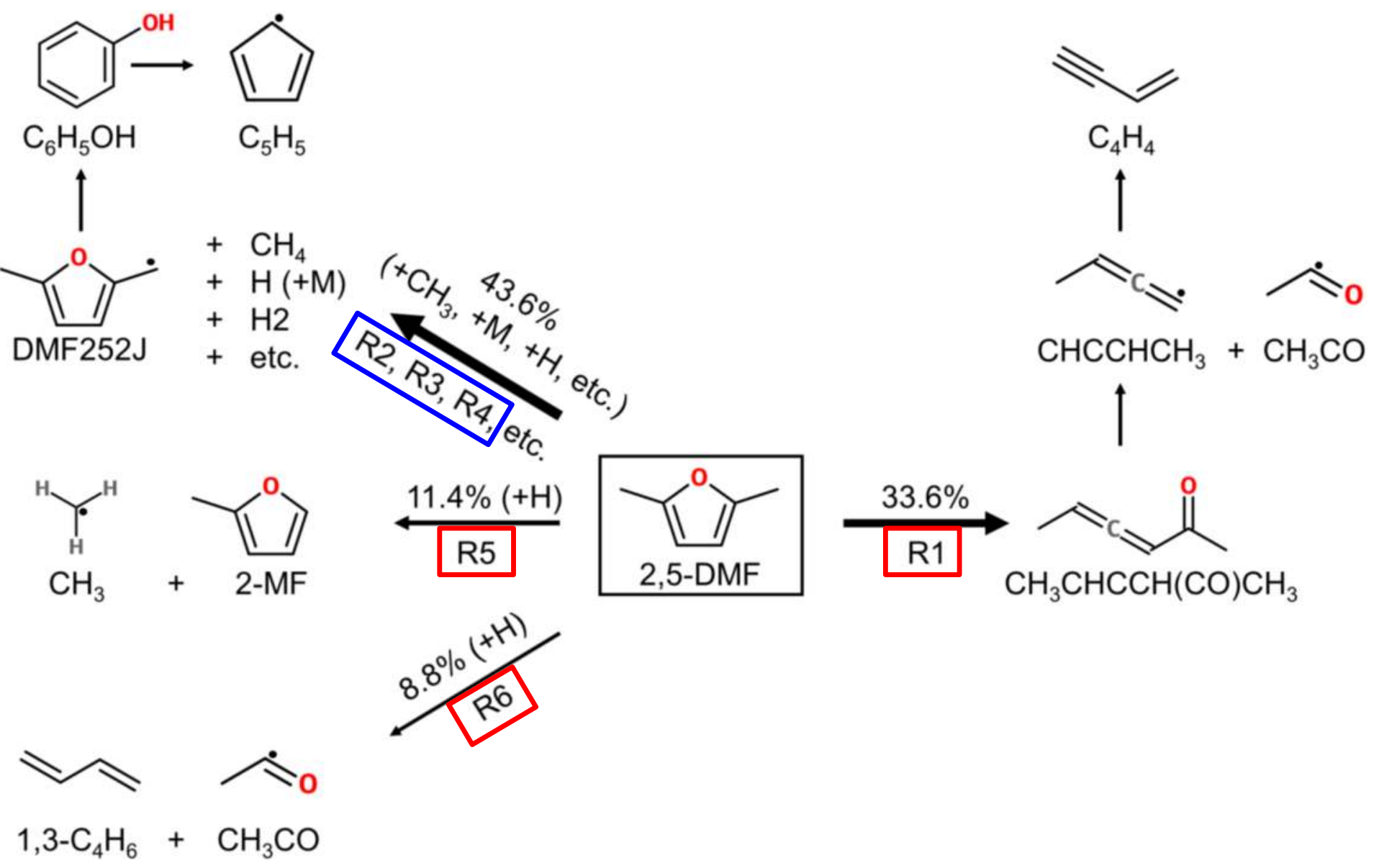

- R2-R4 forming effective soot precursor, $\mathrm{C}_{5} \mathrm{H}_{5}$ : positive $S_{r e l}$

- R1, R5, R6 forming less effective soot precursors: negative $S_{r e l}$ 


\section{Rate Constant Uncertainties on YSI Predictions}

Reference Fuels

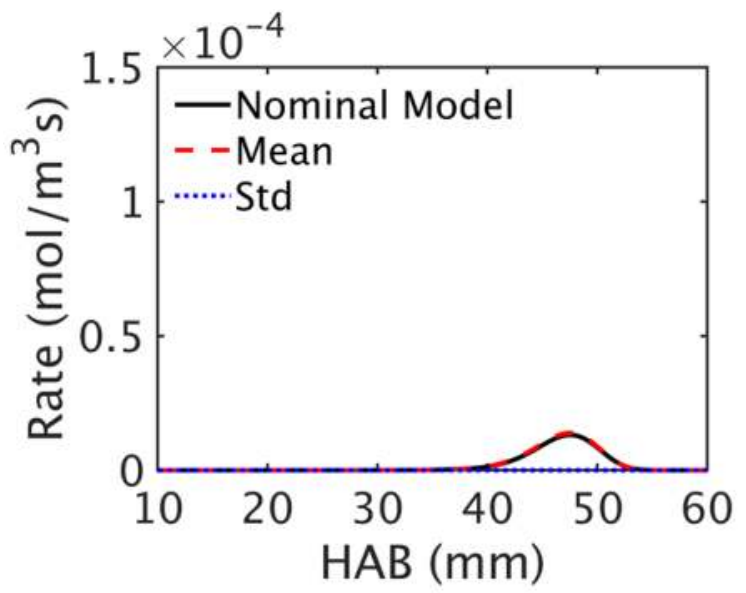

(a) Methanol

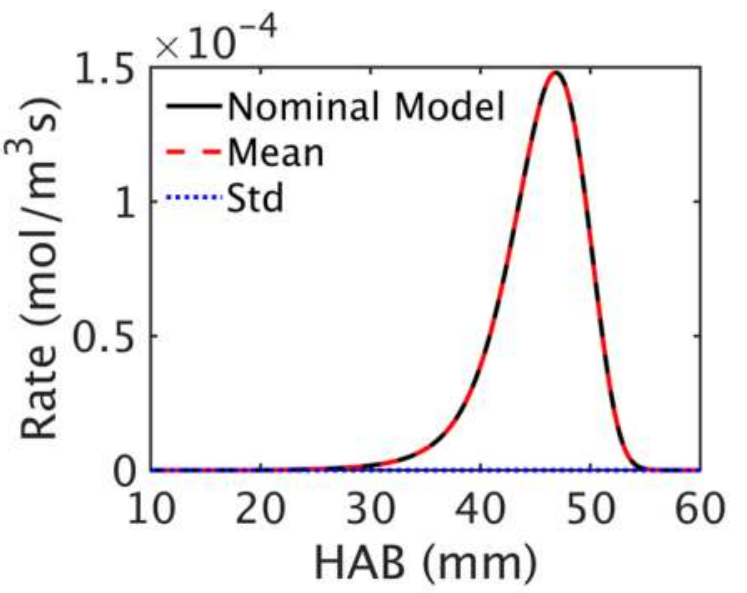

(b) 2,4,4-Trimethyl-2-pentene
Target Fuel

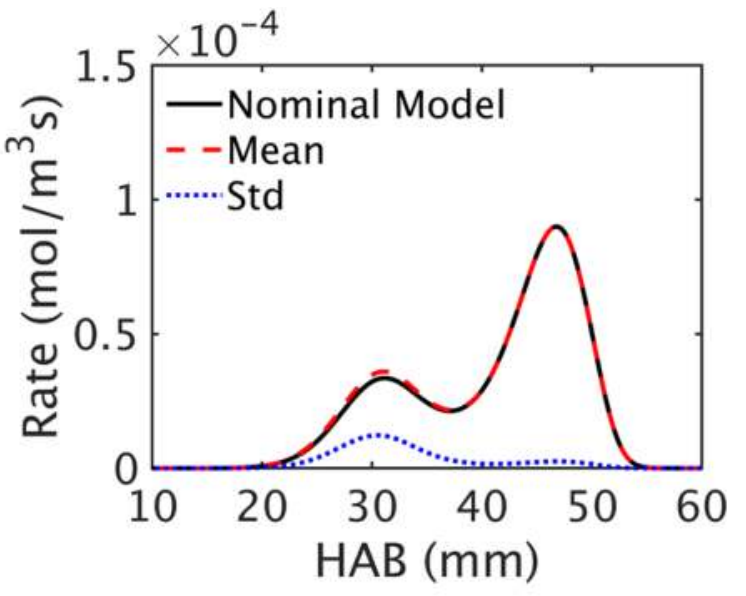

(c) 2,5-DMF

- Randomly perturb the sensitive reaction rates altogether within a factor of 2 [50\%, 200\%]

- 500 randomly perturbed flame simulations

- Re-calculate YSI using the mean soot production rates \pm 2 standard deviations

- Good agreement under the first bullet point

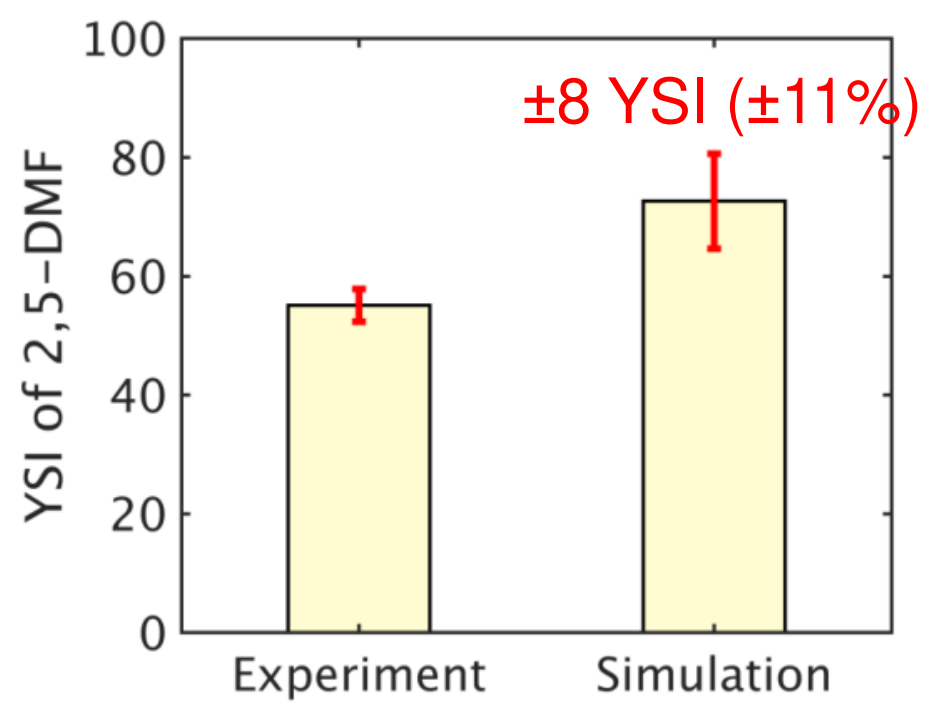


- YSIs of 20 bio-fuels were numerically predicted.

- PAH growth reactions had little impact on YSI.
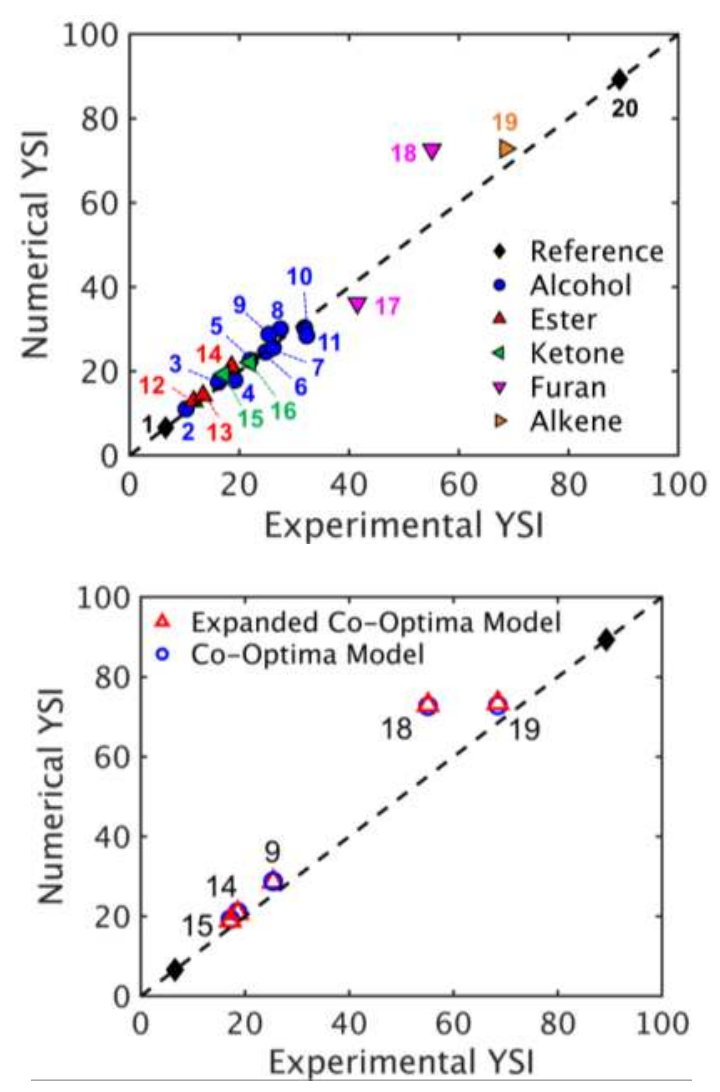

- A modified sensitivity coefficient was introduced.

- Rate constant uncertainties on YSI of 2,5-DMF was quantified.

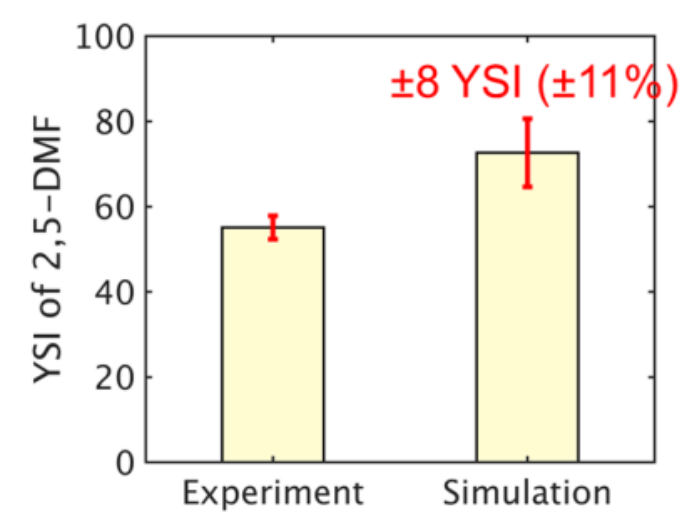

